\title{
First detection of hydrogen isocyanide (HNC) in Titan's atmosphere
}

\author{
R. Moreno ${ }^{1}$, E. Lellouch ${ }^{1}$, L. M. Lara ${ }^{2}$, R. Courtin ${ }^{1}$, D. Bockelée-Morvan ${ }^{1}$, P. Hartogh ${ }^{3}$, \\ M. Rengel ${ }^{3}$, N. Biver ${ }^{1}$, M. Banaszkiewicz ${ }^{4}$, and A. González ${ }^{3,2}$ \\ ${ }^{1}$ LESIA - Observatoire de Paris, CNRS, Université Paris 06, Université Paris-Diderot, 5 place Jules Janssen, 92195 Meudon, France \\ e-mail: raphael.moreno@obspm.fr \\ 2 Instituto de Astrofísica de Andalucía (CSIC), Granada, Spain \\ 3 Max-Planck-Institut für Sonnensystemforschung, Katlenburg-Lindau, Germany \\ ${ }^{4}$ Space Research Centre of Polish Academy of Sciences, Warsaw, Poland \\ Received 30 September 2011 / Accepted 22 November 2011
}

ABSTRACT

\begin{abstract}
We report on the first identification of hydrogen isocyanide (HNC) in Titan's atmosphere, from observations using the HIFI instrument on the Herschel ${ }^{\star}$ Space Observatory. An emission line from the HNC $J=6 \rightarrow 5$ rotational transition at $543.897 \mathrm{GHz}$ was measured in Titan on June 14 and December 31, 2010. Radiative transfer modeling indicates that the bulk of HNC is located above $400 \mathrm{~km}$, with a column density in the range $(0.6-1.5) \times 10^{13} \mathrm{~cm}^{-2}$, but the observations cannot establish its vertical profile. In particular HNC could be restricted to the upper thermosphere $(\sim 1000 \mathrm{~km})$, in which case its local abundance relative to HCN could be as high as $\sim 0.3$. $\mathrm{HNC}$ is probably formed mostly at ionospheric levels $(950-1150 \mathrm{~km})$ from dissociative recombination of $\mathrm{HCNH}^{+}$and possibly other heavier nitrile ions. Ionospheric loss of $\mathrm{HNC}$ occurs by protonation with $\mathrm{XH}^{+}$ions. Additional formation (e.g. from $\left.\mathrm{N}\left({ }^{4} \mathrm{~S}\right)+{ }^{3} \mathrm{CH}_{2}\right)$ and loss routes (e.g. from isomerization to $\mathrm{HCN}$ ) in the neutral atmosphere remain to be investigated.
\end{abstract}

Key words. techniques: spectroscopic - submillimeter: planetary systems - planets and satellites: individual: Titan

\section{Introduction}

Titan is the only satellite of the Solar System to possess a dense atmosphere (1.5 bar), mainly composed of $\mathrm{N}_{2}$ with a few percent of $\mathrm{CH}_{4}$. The detection of hydrogen cyanide $(\mathrm{HCN})$ and of more complex nitriles $\left(\mathrm{HC}_{3} \mathrm{~N}\right.$ and $\left.\mathrm{C}_{2} \mathrm{~N}_{2}\right)$ obtained by IR spectrometers onboard spacecrafts (starting with Voyager in 1980) was of considerable interest, as these molecules are key intermediates in the synthesis of organic molecules. Their presence, along with that of several hydrocarbons, implies a complex photochemistry of methane, which is, on the one hand, coupled with that of $\mathrm{N}_{2}$, and on the other hand, enriched by ion-molecule reactions taking place in the upper atmosphere $(>800 \mathrm{~km})$. As a matter of fact, and as one of the major discoveries of the Cassini mission at Titan, numerous heavy organic molecules have been detected in Titan's upper atmosphere by Cassini Ion Neutral Mass Spectrometer (INMS), (e.g. Waite et al. 2005; Vuitton et al. 2007). Despite these impressive results, from which a completely new view of Titan's ion-neutral chemistry emerged, INMS cannot distinguish between species of identical mass, in particular between isomers. Heterodyne spectroscopy of strong rotational lines provides high sensitivity and frequency discrimination for the detection of minor species with permanent dipole moment - such as $\mathrm{HCN}, \mathrm{HC}_{3} \mathrm{~N}$ and $\mathrm{CH}_{3} \mathrm{CN}$ (Marten et al. 2002; Gurwell 2004), and CO (Gurwell \& Muhleman 1995; Hidayat et al. 1998; Rengel et al. 2011) - and allows for full resolution of line profiles, as well as unique absolute wind measurements in Titan's stratosphere and mesosphere from mapping of the Doppler shift in spatially-resolved data (Moreno et al. 2005).

\footnotetext{
* Herschel is an ESA space observatory with science instruments provided by European-led Principal Investigator consortia and with important participation from NASA.
}

In this paper, we report the first detection of a new species, hydrogen isocyanide (HNC), in Titan's atmosphere, using the Heterodyne Instrument for the Far-Infrared (HIFI, de Graauw et al. 2010) onboard the ESA Herschel Space Observatory (Pilbratt et al. 2010).

\section{Observations}

Observations of Titan were performed on June 14 and December 31, 2010, as part of the Herschel guaranteed time key program "Water and related chemistry in the Solar System" (HssO, see Hartogh et al. 2009). We used the HIFI heterodyne receiver in band $1 \mathrm{a}$ (covering the $480-560 \mathrm{GHz}$ spectral region), with two orthogonal polarizations ( $\mathrm{H}$ and $\mathrm{V}$ ) simultaneously.

The original goal of these observations was to study water vapor in Titan's atmosphere. The receiver was therefore tuned to the $\mathrm{H}_{2} \mathrm{O}\left(1_{10}-1_{01}\right)$ rotational transition at $556.936 \mathrm{GHz}$, in the upper sideband (USB). In June 2010, the local oscillator (LO) frequency of the instrument was tuned to $550.390 \mathrm{GHz}$. Because HIFI is a double sideband (DSB) receiver, its lower sideband (LSB) simultaneously covered the $542.4-546.4 \mathrm{GHz}$ range. For spectral analysis, we used two different spectrometers at a spectral resolution of $1.1 \mathrm{MHz}$ (Wide Band Spectrometer, WBS) and $0.25 \mathrm{MHz}$ (High Resolution Spectrometer, HRS). The telescope half power beam width $(H P B W)$ at $556 \mathrm{GHz}$ is $39^{\prime \prime}$. To minimize the contribution from Saturn, Titan was observed at eastern elongation (173" east of Saturn) using a position switch (PSw) observing mode. The reference sky position was chosen to be separated from Titan by $+2^{\prime}$ in declination (i.e. North of Titan) in order to avoid any contamination from Saturn. Data reduction was carried out using the Herschel data reduction software (HIPE, Ott 2010), which calibrates the data flux and corrects for spacecraft and planet velocity. A polynomial baseline removal was performed to eliminate the standing waves. 


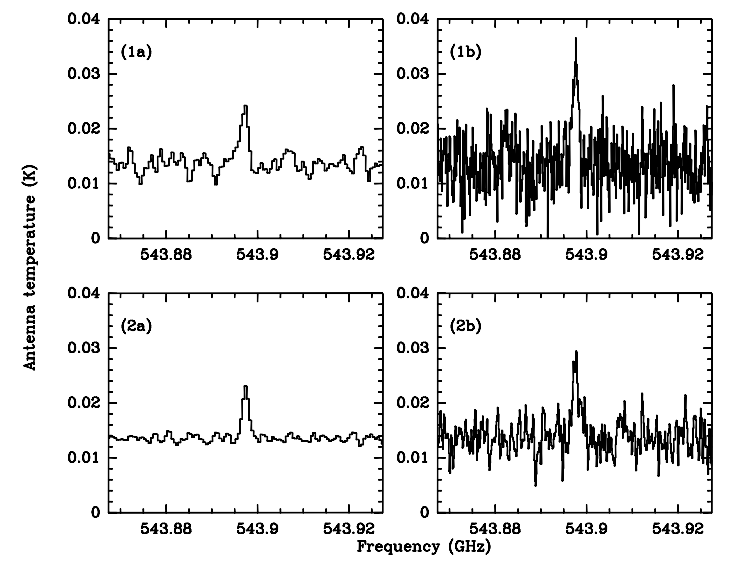

Fig. 1. Observations of $\mathrm{HNC}(6-5)$ at $543.897 \mathrm{GHz}$ on Titan measured with Herschel/HIFI. Both $\mathrm{H}$ and V polarizations are averaged. 1a) Upper left panel: measurement with $\mathrm{WBS}(\Delta v=1.1 \mathrm{MHz})$ on June 14, 2010. 1b) Upper right panel: measurement with HRS $(\Delta v=$ $0.25 \mathrm{MHz}$ ) on June 14, 2010. 2a) Lower left panel: measurement with WBS on Dec. 31, 2010. 2b) Lower right panel: measurement with HRS on Dec. 31, 2010. Due to varying distance of Titan, observations on Dec. 31 are scaled in intensity to the conditions of June 14 .

Table 1. Summary of HIFI observations of Titan.

\begin{tabular}{lcccc}
\hline \hline $\begin{array}{l}\text { UT start date } \\
\text { [yyyy mm dd.ddd] }\end{array}$ & $\begin{array}{c}\text { Integration } \\
\text { time [h] }\end{array}$ & $\begin{array}{c}\text { Obs. } \\
\text { mode }\end{array}$ & $\begin{array}{c}\Delta \\
{[\mathrm{AU}]}\end{array}$ & $\begin{array}{c}\theta \\
{\left[{ }^{\prime \prime}\right]}\end{array}$ \\
\hline 20100614.822 & 4.54 & PSw & 9.390 & 0.756 \\
20101231.321 & 3.62 & FSw & 9.661 & 0.735 \\
\hline
\end{tabular}

Notes. $\Delta$ is the distance between Titan and Herschel. $\theta$ is the surface apparent diameter. Observing modes are described in the text.

In addition to the water line (which will be reported separately), we unexpectedly detected in the LSB a narrow emission line at $543.897 \mathrm{GHz}$ (Fig. 1), which we identified as the $\mathrm{HNC}(6-5)$ transition, representing the first detection of $\mathrm{HNC}$ in Titan's atmosphere. To confirm the detection, additional observations of Titan were obtained on December 31, 2010, this time at western elongation. To increase the signal-to-noise ratio $(\mathrm{S} / \mathrm{N})$, instead of position switch we used the standard frequency switch (FSw) observing mode, with a frequency throw of 94.5 MHz. To confirm that the line was indeed from HNC(6-5), we used a slightly different tuning, 1 changing the LO frequency to $550.325 \mathrm{GHz}$. The line was again unambiguously detected, separately on each of the two spectrometers (Fig. 1) at the frequency of HNC(6-5). For both observational epochs, the HNC emission is narrow ( $F W H M \sim 0.8 \mathrm{~km} \mathrm{~s}^{-1}$, or $\left.1.5 \mathrm{MHz}\right)$. The apparent diameter of Titan on December 31 was $\sim 6 \%$ smaller than on June 14 (Table 1). After scaling the HNC line intensity to the June 14 conditions (Fig. 1), the line intensity ratio between the two epochs (June/Dec.) is $1.02 \pm 0.10$ implying no significant intensity variation over this time period or between the leading and trailing sides.

\section{Modeling}

The HNC spectra were analyzed with a line-by-line radiative transfer code accounting for the spherical geometry of Titan's atmosphere (Marten et al. 2002, and references therein). This model considers HNC molecular opacities from the JPL catalog (Pickett et al. 1998), and also includes collision-induced opacities of $\mathrm{N}_{2}-\mathrm{N}_{2}, \mathrm{~N}_{2}-\mathrm{CH}_{4}$, and $\mathrm{CH}_{4}-\mathrm{CH}_{4}$ (Borysow \& Frommhold 1986, 1987; Borysow \& Tang 1993). The pressure broadening coefficient $\left(\gamma_{0}\right)$ of $\mathrm{HNC}(6-5)$ was taken to be equal to that of $\mathrm{HCN}(6-5), \gamma_{0}=0.129 \mathrm{~cm}^{-1} \mathrm{bar}^{-1}$ at a reference temperature of $300 \mathrm{~K}$ and with a temperature dependency exponent $n=0.69$ (Yang et al. 2008). The thermal profile used for our computation is a combination of (i) the temperatures measured by Huygens/HASI (Fulchignoni et al. 2005) in the troposphere at altitudes between 0-140 km; (ii) the disk-averaged Cassini/CIRS stratospheric temperatures (Vinatier et al. 2010) at altitudes between 140-500 km; (iii) the Cassini/INMS retrieved temperatures (i.e. $155 \mathrm{~K}$ in average, De La Haye et al. 2007) at altitudes between 1000-1500 km; (iv) a decreasing temperature from $165 \mathrm{~K}$ to $155 \mathrm{~K}$ at altitudes between 500 and $1000 \mathrm{~km}$. Our modeling results, presented below, indicate that the $\mathrm{HNC}(6-5)$ line is optically thin (opacity $\tau \sim 0.12$ ), therefore results are not too sensitive to the precise temperature profile.

The model computes spectra in units of fluxes or RayleighJeans temperatures $\left(T_{\mathrm{rj}}\right)$. To compare $T_{\mathrm{rj}}$ with the measured antenna temperature $\left(T_{\mathrm{a}}\right)$, we use the usual relationship: $T_{\mathrm{a}}=$ $T_{\mathrm{rj}} / F_{\mathrm{d}} \times B_{\text {eff }} / F_{\text {eff }}$, with $F_{\mathrm{d}}$ the geometrical dilution factor, $B_{\text {eff }}$ the telescope beam efficiency (i.e. 0.75 ), and $F_{\text {eff }}$ the telescope forward efficiency (i.e. 0.96). Because the HRS spectra are much noisier (due to their higher spectral resolution) than the WBS data, we focussed on the WBS spectra, averaging the two epochs to further improve the $\mathrm{S} / \mathrm{N}$.

\subsection{Uniform mixing ratio profiles}

The HNC narrow linewidth indicates that the line is mostly Doppler-broadened, with little or no contribution from Lorentz linewings. In a first series of models, we considered uniform vertical distributions of $\mathrm{HNC}$, i.e. with a constant mixing ratio $q_{0}$ above a given altitude $z_{0}$. In this approach, one can discard HNC profiles with $z_{0}$ smaller than $200 \mathrm{~km}$, because the associated simulated lines are broader than the observed line (i.e. pressurebroadened, Fig. 2). While a value of $z_{0}$ equal to $300 \mathrm{~km}$ is still acceptable, we favor uniform profiles in which $z_{0}$ is greater than $400 \mathrm{~km}$. This, in particular, is also consistent with HNC being restricted to the upper thermosphere (e.g. $z_{0}=1000 \mathrm{~km}$ ). For such uniform distributions characterized by $z_{0}$, the probed altitudes mainly lie between $z_{0}$ and $z_{0}+100 \mathrm{~km}$. Best-fit results of the coupled parameters $z_{0}$ and $q_{0}$ are given in Table 2. The retrieved column density depends slightly on $z_{0}$, and lies in the range $(0.6-1.5) \times 10^{13} \mathrm{~cm}^{-2}$ for $z_{0}$ varying between 400 and $1000 \mathrm{~km}$, with higher values of $z_{0}$ associated with smaller columns.

\subsection{A thermospheric case}

As illustrated above, our observations do not establish the vertical distribution of HNC, except for the loose constraint that it cannot be present in large amounts below $300 \mathrm{~km}$. Nevertheless, as we discuss below, formation scenarios argue for a large fraction of HNC to be restricted to the thermosphere, with its distribution roughly following that of the electronic density in the lower ionosphere. To mimick this situation, we tested the case in which HNC is restricted to a layer from 900 to $1200 \mathrm{~km}$, with a constant number density (i.e. a mixing ratio $(q)$ increasing with altitude as $q=q_{0} \times\left(p_{0} / p\right)$, with $p_{0}$ the reference pressure corresponding to $\left.z_{0}=900 \mathrm{~km}\right)$. For altitudes lower than $\mathrm{z}_{0}$, the mixing ratio is taken as zero. Taking into account the high molecular diffusion above the homopause at $\sim 1200 \mathrm{~km}$ $\left(D=0.5--1 \times 10^{10} \mathrm{~cm}^{2} \mathrm{~s}^{-1}\right.$, De La Haye et al. 2007; Yelle et al. 2008), we also assumed a constant mixing ratio above the homopause $z_{\mathrm{h}}=1200 \mathrm{~km}$. With this model, the best fit is obtained 
R. Moreno et al.: First detection of HNC in Titan's atmosphere

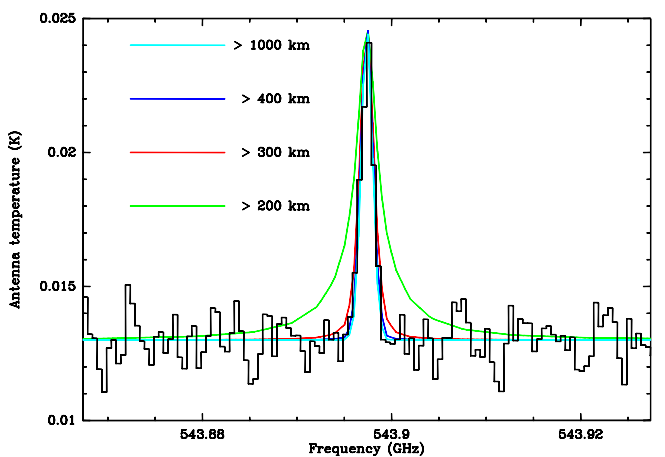

Fig. 2. Models of the $\mathrm{HNC}(6-5)$ line, assuming an uniform mixing ratio distribution of HNC above altitudes of $1000,400,300$, and $200 \mathrm{~km}$, corresponding to cases A, G, H, and I, respectively, in Table 2. These models are compared with the averaged WBS spectrum from June/Dec.

for $q_{0}(900 \mathrm{~km})=\left(2.0_{-0.4}^{+0.4}\right) \times 10^{-6}$. It corresponds to an $\mathrm{HNC}$ column density of $5.7_{-1.1}^{+1.1} \times 10^{12} \mathrm{~cm}^{-2}$, close to the one retrieved for uniform $A$ or $B$ cases (Table 2). Extending this approach to other values of $z_{0}$, we also computed models with increasing mixing ratio with altitude as $q=q_{0} \times\left(p_{0} / p\right)^{n}$. Many possible fits were found for $z_{0}$ between $300-1000 \mathrm{~km}$, further demonstrating that we cannot currently constrain the vertical distribution of HNC. The retrieved column density remains unchanged at $(0.6-1.5) \times 10^{13} \mathrm{~cm}^{-2}$.

\section{Discussion}

The possible presence of HNC in Titan's atmosphere was first considered by Petrie (2001). Based on pre-Cassini photochemical models (e.g. Ip 1990; Galand et al. 1999; Banaszkiewicz et al. 2000) which predicted that $\mathrm{HCNH}^{+}$is one important or even the dominant ion at the ionospheric peak, Petrie (2001) postulates the dissociative recombination of $\mathrm{HCNH}^{+}\left(\mathrm{HCNH}^{+}+\mathrm{e}^{-} \rightarrow\right.$ $\mathrm{HNC}+\mathrm{H})$ as an important source of HNC. This reaction, which produces $\mathrm{HCN}$ and $\mathrm{HNC}$ in approximately equal yields, was suggested as the origin of the high abundance of HNC $(\mathrm{HNC} \sim \mathrm{HCN})$ in dense molecular clouds as early as 35 years ago (Watson 1976). Petrie (2001) further considered various loss processes for HNC, including (i) photon-induced isomerization $(\mathrm{HNC}+\mathrm{h} v \rightarrow \mathrm{HCN})$; (ii) photolysis $(\mathrm{HNC}+\mathrm{h} v \rightarrow \mathrm{H}+$ $\mathrm{CN}$ ); (iii) $\mathrm{H}$-catalyzed isomerization $(\mathrm{HNC}+\mathrm{H} \rightarrow \mathrm{HCN}+\mathrm{H})$; (iv) protonation with ionospheric cations $\left(\mathrm{XH}^{+}+\mathrm{HNC} \rightarrow \mathrm{X}+\right.$ $\left.\mathrm{HCNH}^{+}\right)$; and (v) reactions with neutral radicals $(\mathrm{X}+\mathrm{HNC} \rightarrow$ $\mathrm{XCN}+\mathrm{H}$ or $\mathrm{XH}+\mathrm{CN}$ ). He dismissed (i) and (ii) as being insignificant compared to protonation and retained the other three loss mechanisms, focussing on process $(v)$ with $\mathrm{X}=\mathrm{CH}_{3}$. Using this limited chemistry, HNC would therefore result from the following sets of reactions:

$$
\begin{aligned}
\mathrm{HCNH}^{+}+\mathrm{e}^{-} & \rightarrow \mathrm{HNC}+\mathrm{H} \\
& \rightarrow \mathrm{HCN}+\mathrm{H} \\
\mathrm{XH}^{+}+\mathrm{HNC} & \rightarrow \mathrm{X}+\mathrm{HCNH}^{+} \\
\mathrm{HNC}+\mathrm{H} & \rightarrow \mathrm{HCN}+\mathrm{H} \\
\mathrm{CH}_{3}+\mathrm{HNC} & \rightarrow \mathrm{CH}_{3} \mathrm{CN}+\mathrm{H} \\
& \rightarrow \mathrm{CH}_{4}+\mathrm{CN}
\end{aligned}
$$

The detection of $\mathrm{HCNH}^{+}$by INMS with a peak abundance of $\sim 10^{3} \mathrm{~cm}^{-3}$ near 1100-1150 km (e.g. Waite et al. 2005; Vuitton et al. 2007; Cui et al. 2009) supports the above production scheme and allows one to evaluate it quantitatively, although key reaction rates $\left(k_{i}\right)$ are still uncertain. The dissociative recombination rate of $\mathrm{HCNH}^{+}$depends on the electron temperature. The
Table 2. Retrieved HNC mixing ratio and column density assuming uniform profiles above altitude $z_{0}$.

\begin{tabular}{lccc}
\hline \hline Profile & $\geq z_{0}(\mathrm{~km})$ & Mixing ratio & Column $\left(\mathrm{cm}^{-2}\right)$ \\
\hline A & 1000 & $6.0_{-1.0}^{+1.5} \times 10^{-5}$ & $6.3 \times 10^{12}$ \\
B & 900 & $1.4_{-0.3}^{+0.3} \times 10^{-5}$ & $6.9 \times 10^{12}$ \\
C & 800 & $3.1_{-0.6}^{+0.8} \times 10^{-6}$ & $7.8 \times 10^{12}$ \\
D & 700 & $6.7_{-1.2}^{+1.8} \times 10^{-7}$ & $8.9 \times 10^{12}$ \\
E & 600 & $1.3_{-0.3}^{+0.3} \times 10^{-7}$ & $1.0 \times 10^{13}$ \\
F & 500 & $2.6_{-0.5}^{+0.8} \times 10^{-8}$ & $1.2 \times 10^{13}$ \\
G & 400 & $4.5_{-1.0}^{+1.2} \times 10^{-9}$ & $1.5 \times 10^{13}$ \\
H & $300^{a}$ & $1.1_{-0.3}^{+0.3} \times 10^{-9}$ & $2.0 \times 10^{13}$ \\
I & $200^{b}$ & $0.4_{-0.1}^{+0.1} \times 10^{-9}$ & - \\
\hline
\end{tabular}

Notes. Mixing ratio include uncertainties in HIFI calibration $(\sim 10 \%$, Roelfsema et al. 2011) and statistical uncertainties. ${ }^{(a)}$ Linewidth is marginally too large. ${ }^{(b)}$ Linewidth is too large.

Cassini Radio and Plasma Wave Science (RPWS/LP) measurements have reported an electronic temperature of $500 \mathrm{~K}$ ( ̊gren et al. 2009; Galand et al. 2010) over 950-1100 km. Such a value is surprisingly high, because electronic temperatures closer to the $\sim 150 \mathrm{~K}$ gas temperature are expected below $\sim 1050 \mathrm{~km}$ (Richards et al. 2011). We therefore considered both $T_{\mathrm{e}}=150 \mathrm{~K}$ and $T_{\mathrm{e}}=500 \mathrm{~K}$, giving $k_{1 \mathrm{a}}=k_{1 \mathrm{~b}}=1.5 \times 10^{-7} \mathrm{~cm}^{3} \mathrm{~s}^{-1}$ and $6.90 \times 10^{-8} \mathrm{~cm}^{3} \mathrm{~s}^{-1}$, respectively (Semaniak et al. 2001).

As discussed by Petrie (2001), protonation is likely to involve virtually all hydrogen-bearing ions, so it is reasonable to adopt a total $\mathrm{XH}^{+}$concentration equal to the electron density. In particular, the $\mathrm{HCNH}^{+}+\mathrm{HNC}$ reaction has been investigated theoretically, and suggested to be a significant loss channel for HNC in molecular clouds (Pichierri 2002). To our knowledge however, no measurements of the protonation rates for $\mathrm{HNC}$ are available. Petrie uses $k_{2}=3.5 \times 10^{-9} \mathrm{~cm}^{3} \mathrm{~s}^{-1}$. The Su-Chesnavich formulae (see Woon \& Herbst 2009) would give $k_{2}=5.2 \times 10^{-9} \mathrm{~cm}^{3} \mathrm{~s}^{-1}$ at $150 \mathrm{~K}$. As this value seems high compared to typical protonation rates (Vigren, priv. comm.), we here considered $k_{2}=3.5 \times 10^{-9} \mathrm{~cm}^{3} \mathrm{~s}^{-1}$ and $k_{2}=1 \times 10^{-9} \mathrm{~cm}^{3} \mathrm{~s}^{-1}$. With a peak electron density of $\sim 2000 \mathrm{~cm}^{-3}$ (Wahlund et al. $2009)$, this gives a loss rate of $(2-7) \times 10^{-6} \mathrm{~s}^{-1}$.

Following Talbi et al. (1996) we adopt a $k_{3}=1 \times$ $10^{-14} \mathrm{~cm}^{3} \mathrm{~s}^{-1}$ reaction rate (at $150 \mathrm{~K}$ ) for the H-catalyzed isomerization reaction (3). Based on various models (e.g. Lara et al. 1996; De La Haye et al. 2008), the [H] mixing ratio near $1100 \mathrm{~km}$ is in the range $(4-10) \times 10^{-4}$. Adopting $4 \times 10^{-4}$ from De La Haye et al. (2008), which gives $[\mathrm{H}] \sim 1.3 \times 10^{6} \mathrm{~cm}^{-3}$ at $1100 \mathrm{~km}$, implies a loss rate of $\sim 1.3 \times 10^{-8} \mathrm{~s}^{-1}$ through reaction (3), much lower than the above losses from ion-neutral reactions. Regarding the $\mathrm{CH}_{3}+\mathrm{HNC}$ (4) reaction rate, Petrie (2001) considered two extreme values $\left(k_{4}=10^{-14}\right.$ and $k_{4}=$ $\left.5 \times 10^{-11} \mathrm{~cm}^{3} \mathrm{~s}^{-1}\right)$, but in a more recent study, Petrie \& Osamura (2004) indicate a much lower rate $\left(9.2 \times 10^{-20} \mathrm{~cm}^{3} \mathrm{~s}^{-1}\right.$ at $\left.200 \mathrm{~K}\right)$. With this value and a $\mathrm{CH}_{3}$ concentration of $\sim 2 \times 10^{6} \mathrm{~cm}^{-3}$ at $1100 \mathrm{~km}$ (e.g. Hörst et al. 2008), the associated loss is entirely insignificant, and the HNC abundance is thus controlled by reactions (1a) and (2).

With the above scenario, the chemical lifetime of $\mathrm{HNC}$ is $(1.4-5) \times 10^{5}$ s, i.e. 3 to 10 times shorter than Titan's day and comparable to the day-to-night transport time (about $2 \times 10^{5} \mathrm{~s}$ for $\sim 50 \mathrm{~m} / \mathrm{s}$ thermospheric winds, Müller-Wodarg et al. 2008). $\mathrm{HCNH}^{+}$shows strong diurnal variations with maximum (dayside) concentrations of $\sim 1000 \mathrm{~cm}^{-3}$ at $1000-1200 \mathrm{~km}$ (Cui et al. 2009), and about five times less on the nightside, implying that (unobservable so far) diurnal variations of HNC can be expected. The HNC chemical lifetime is comparable to the 
transport timescale $\left(1.5 \times 10^{5} \mathrm{~s}\right.$ at $1000 \mathrm{~km}$, for a $\sim 3 \times 10^{8} \mathrm{~cm}^{2} \mathrm{~s}^{-1}$ diffusion coefficient, Yelle et al. 2008) As a result, HNC in the ionosphere is affected by both chemical and transport losses, but its distribution may be reasonably close to photochemical equilibrium. In this framework, and considering only the protonation loss with $\left[\mathrm{XH}^{+}\right]=\left[\mathrm{e}^{-}\right]$, the $\mathrm{HNC}$ concentration can be simply written as $[\mathrm{HNC}]=k_{1 a} / k_{2} \times\left[\mathrm{HCNH}^{+}\right]$, where the equality holds either for concentrations or column densities. With an $\mathrm{HCNH}^{+}$dayside (relevant for our purpose) column density of $3.5 \times 10^{10} \mathrm{~cm}^{-2}$, and using each of these two values considered for $k_{1 a}$ and for $k_{2}$, we obtain an HNC column of $7 \times 10^{11}$ $5.2 \times 10^{12} \mathrm{~cm}^{-2}$. The upper range is consistent with the measurements, although somewhat marginally.

The above calculations only consider HNC production from $\mathrm{HCNH}^{+}$, while any other ion that includes the $-\mathrm{CNH}^{+}$group may lead to $\mathrm{HNC}$ upon recombination. $\mathrm{HCNH}^{+}$is the dominant ion measured by INMS, followed by $\mathrm{C}_{2} \mathrm{H}_{5}{ }^{+}, \mathrm{HC}_{3} \mathrm{NH}^{+}$, c- $\mathrm{C}_{3} \mathrm{H}_{3}{ }^{+}, \mathrm{C}_{3} \mathrm{H}_{5}{ }^{+}$(Cui et al. 2009), but RPWS and the Cassini Plasma Spectrometer (CAPS) measurements indicate that heavy ions beyond the mass range of INMS (>100 amu) contribute significantly, constituting $~ 5 \%$ of the ionosphere near $1100 \mathrm{~km}$ and becoming even dominant (50-70\%) over 950-1000 km altitude (Crary et al. 2009; Wahlund et al. 2009). Nitrile ions heavier than $\mathrm{HCNH}^{+}$have higher recombination rates, e.g. by factors 3 to 5 for $\mathrm{HC}_{3} \mathrm{NH}^{+}$and other species measured by Vigren et al. (2011). Remarkably, "effective" recombination rates for Titan's ionosphere, based on CAPS and RPWS/LP measurements (Galand et al. 2010), increase below $1200 \mathrm{~km}$, reaching $\sim 5 \times 10^{-6} \mathrm{~cm}^{3} \mathrm{~s}^{-1}$ at $1000 \mathrm{~km}$, a behavior attributed to the change of composition below the ionospheric peak and the progressive onset of heavy ions. Since heavy nitrile ions in this altitude range could provide significant additional sources of $\mathrm{HNC}$, we conclude that a purely ionospheric source may be quantitatively viable for $\mathrm{HNC}$, provided the protonation rates are not too high.

Even if a primarily ionospheric production is assumed, HNC will not necessarily be restricted to the ionosphere, as it must be transported downward to some extent by eddy mixing. This will further increase its chemical lifetime, in relation to the decline of ion-molecule reactions. Petrie \& Osamura (2004) find that in the neutral atmosphere, H-catalyzed isomerization (3) may be the main loss channel for $\mathrm{HNC}$, with additional contributions from reactions with $\mathrm{CN}$ and $\mathrm{C}_{3} \mathrm{~N}$. Photolysis and photo-isomerization of HNC, albeit negligible at ionospheric levels, must also become significant there, especially the latter, which has a very low energy threshold ( $8510 \AA$ A, see Petrie 2001). Additional formation routes to $\mathrm{HNC}$ in the neutral atmosphere should also be investigated, in particular its production through $\mathrm{N}\left({ }^{4} \mathrm{~S}\right)+{ }^{3} \mathrm{CH}_{2}$. This pathway produces equal amounts of HCN and HNC and has been invoked in the regulation of the $\mathrm{HNC} / \mathrm{HCN}$ abundance in molecular clouds (Herbst et al. 2000). We leave these issues for future photochemical modeling, with the goal of matching HNC and HCN simultaneously. We note that, in the model where HNC is restricted to altitudes above $1000 \mathrm{~km}$, its mole fraction is $\sim 6 \times 10^{-5}$. Comparing with the HCN abundance from INMS $\left(2.0 \times 10^{-4}\right.$, Vuitton et al. 2007) indicates $\mathrm{HNC} / \mathrm{HCN} \sim 0.3$; thus, a significant contribution of HNC to the mass 27 signal is likely.

In the Solar System, HNC has been observed in many comets since its first discovery in comet Hyakutake, with $\mathrm{HNC} / \mathrm{HCN}$ abundance ratios varying from $\leq 2 \%$ to $20 \%$ (Irvine et al. 1996; Lis et al. 2008). The inverse correlation of this ratio with heliocentric distance argues for the production of HNC from the thermal degradation of organic grains heated by the Sun (Lis et al. 2008). A production of HNC from solid (haze) material in Titan's atmosphere might therefore not be excluded, although this remains largely speculative. In this respect, and more generally to constrain photochemical models, determining the vertical profile of $\mathrm{HNC}$ and its abundance in the region of the main haze $(300-500 \mathrm{~km})$ would be important. Although observing other (and stronger) additional lines of HNC might help slightly, this will probably require limb observations from a Titan orbiter equipped with a submillimeter instrument (Lellouch et al. 2010).

Acknowledgements. HIFI has been designed and built by a consortium of institutes and university departments from across Europe, Canada, and the United States under the leadership of SRON Netherlands Institute for Space Research, Groningen, The Netherlands, and with major contributions from Germany, France, and the US. We acknowledge very useful discussions with M. Galand, E. Vigren, E. Herbst, S. Petrie and O. Dutuit. L.M. Lara's work has been supported by the Ministry of Innovation and Science through the project AyA 200908011.

\section{References}

Ågren, K., Wahlund, J.-E., Garnier, P., et al. 2009, Planet. Space Sci., 57, 1821 Banaszkiewicz, M., Lara, L. M., Rodrigo, R., López-Moreno, J. J., \& MolinaCuberos, G. J. 2000, Icarus, 147, 386

Borysow, A., \& Frommhold, L. 1986, ApJ, 311, 1043

Borysow, A., \& Frommhold, L. 1987, ApJ, 318, 940

Borysow, A., \& Tang, C. 1993, Icarus, 105, 175

Crary, F. J., Magee, B. A., Mandt, K., et al. 2009, Planet. Space Sci., 57, 1847

Cui, J., Galand, M., Yelle, R. V., et al. 2009, J. Geophys. Res., A, 114, A06310 de Graauw, T., Helmich, F. P., Phillips, T. G., et al. 2010, A\&A, 518, L6

De La Haye, V., Waite, J. H., Johnson, R. E., et al. 2007, J. Geophys. Res. A, 112, A07309

De La Haye, V., Waite, J. H., Cravens, T. E., Robertson, I. P., \& Lebonnois, S. 2008, Icarus, 197, 110

Fulchignoni, M., Ferri, F., Angrilli, F., et al. 2005, Nature, 438, 785

Galand, M., Lilensten, J., Toublanc, D., \& Maurice, S. 1999, Icarus, 140, 92

Galand, M., Yelle, R., Cui, J., et al. 2010, J. Geophys. Res. A, 115, A07312

Gurwell, M. A. 2004, ApJ, 616, L7

Gurwell, M. A., \& Muhleman, D. O. 1995, Icarus, 117, 375

Hartogh, P., Lellouch, E., Crovisier, J., et al. 2009, Planet. Space Sci., 57, 1596

Herbst, E., Terzieva, R., \& Talbi, D. 2000, MNRAS, 311, 869

Hidayat, T., Marten, A., Bezard, B., et al. 1998, Icarus, 133, 109

Hörst, S. M., Vuitton, V., \& Yelle, R. V. 2008, J. Geophys. Res., E, 113, E10006 Ip, W.-H. 1990, ApJ, 362, 354

Irvine, W. M., Bockelee-Morvan, D., Lis, D. C., et al. 1996, Nature, 383, 418

Lara, L. M., Lellouch, E., López-Moreno, J. J., \& Rodrigo, R. 1996, J. Geophys. Res., E, 101, 23261

Lellouch, E., Vinatier, S., Moreno, R., et al. 2010, Planet. Space Sci., 58, 1724

Lis, D. C., Bockelée-Morvan, D., Boissier, J., et al. 2008, ApJ, 675, 931

Marten, A., Hidayat, T., Biraud, Y., \& Moreno, R. 2002, Icarus, 158, 532

Moreno, R., Marten, A., \& Hidayat, T. 2005, A\&A, 437, 319

Müller-Wodarg, I. C. F., Yelle, R. V., Cui, J., \& Waite, J. H. 2008, J. Geophys. Res. (Planets), 113, 10005

Ott, S. 2010, in Astronomical Data Analysis Software and Systems XIX, ed. Y. Mizumoto, K.-I. Morita, \& M. Ohishi, ASP Conf. Ser., 434, 139

Petrie, S. 2001, Icarus, 151, 196

Petrie, S., \& Osamura, Y. 2004, J. Chem. Phys. A, 108, 3623

Pickett, H. M., Poynter, R. L., Cohen, E. A., et al. 1998, J. Quant. Spec. Radiat. Transf., 60, 883

Pilbratt, G. L., Riedinger, J. R., Passvogel, T., et al. 2010, A\&A, 518, L1

Rengel, M., Sagawa, H., \& Hartogh, P. 2011, in World Scientific, Singapore, ed. A. Bhardwaj, Adv. Geosci., 25, 173

Richards, M. S., Cravens, T. E., Robertson, I., et al. 2011, J. Geophys. Res. A, in press

Roelfsema, P. R., Helmich, F. P., Teyssier, D., et al. 2011, A\&A, in press, DOI: 10.1051/0004-6361/201015120

Semaniak, J., Minaev, B. F., Derkatch, A. M., et al. 2001, ApJS, 135, 275

Talbi, D., Ellinger, Y., \& Herbst, E. 1996, A\&A, 314, 688

Vigren, E., Semaniak, J., Hamberg, M., et al. 2011, Planet. Space Sci., in press

Vinatier, S., Bézard, B., Nixon, C. A., et al. 2010, Icarus, 205, 559

Vuitton, V., Yelle, R. V., \& McEwan, M. J. 2007, Icarus, 191, 722

Wahlund, J.-E., Galand, M., Müller-Wodarg, I., et al. 2009, Planet. Space Sci., 57,1857

Waite, J. H., Niemann, H., Yelle, R. V., et al. 2005, Science, 308, 982

Watson, W. D. 1976, Rev. Mod. Phys., 48, 513

Woon, D. E., \& Herbst, E. 2009, ApJS, 185, 273

Yang, C., Buldyreva, J., Gordon, I. E., et al. 2008, J. Quant. Spec. Radiat. Transf., 109,2857

Yelle, R. V., Cui, J., \& Müller-Wodarg, I. C. F. 2008, J. Geophys. Res. E, 113, E10003 